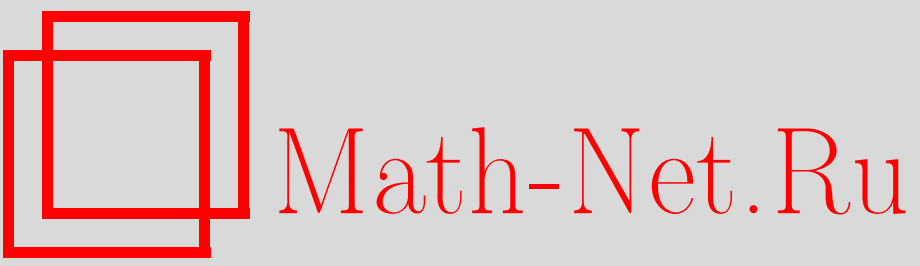

П. Г. Гриневич, С. П. Новиков, Вещественные конечнозонные решения уравнения Sine-Gordon: формула для топологического заряда, УМН, 2001, том 56, выпуск 5, 181-182

DOI: https://doi.org/10.4213/rm443

Использование Общероссийского математического портала Math-Net.Ru подразумевает, что вы прочитали и согласны с пользовательским соглашением

http: //www . mathnet.ru/rus/agreement

Параметры загрузки:

IP : 3.81 .55 .215

26 апреля 2023 г., 15:13:17 


\title{
ВЕЩЕСТВЕННЫЕ КОНЕЧНОЗОННЫЕ РЕШЕНИЯ УРАВНЕНИЯ SINE-GORDON: ФОРМУЛА ДЛЯ ТОПОЛОГИЧЕСКОГО ЗАРЯДА
}

\author{
П. Г. Гриневич, С. П. Новиков
}

Решение уравнения SG $4\left(u_{t t}-u_{x x}\right)=u_{\eta \xi}=4 \sin u$ назьвается периодическим по $x$, если величина $e^{i u}$ периодична. Топологическим зарядом называется целое число $n=[u(x+T)-$ $u(x)] / 2 \pi$, где $T$ - период. Плотность топологического заряда определяется как отношение $\bar{n}=n / T$. Эта величина корректно определена и для квазипериодических функций. Конечнозонные решения SG определяются в терминах алгебраической кривой $\Gamma \mu^{2}=\prod_{k=0}^{2 g}\left(\lambda-E_{k}\right)$, где $E_{0}=0$. Эти решения вещественны и неособы, если точки ветвления $E_{k}$ либо вещественны и отрицательны, $E_{j}<0, j=1, \ldots, 2 m$, либо образуют комплексно-сопряженные пары $\bar{E}_{2 m+2 j-1}=E_{2 m+2 j}, j=1, \ldots, g-m$; при этом дивизор $D+\tau D$ должен быть дивизором нулей мероморфного дифференциала $\Omega$ с полюсами первого порядка только в точках $(0, \infty)$, где $\tau(\lambda, \mu)=(\bar{\lambda}, \bar{\mu})$.

Лемма 1. Дифференциал $\Omega$ имеет вид

$$
\Omega=\left(1-\lambda P_{g-1}(\lambda) R(\lambda)^{-1 / 2}\right) d \lambda,
$$

где полином $P_{g-1}(\lambda)$ - вещественный и такой, что уравнение $R(\lambda)^{-1 / 2}=\lambda P_{g-1}(\lambda)$ имеет точки $\left[(0,0), \gamma_{1}, \ldots, \gamma_{g}, \tau \gamma_{1}, \ldots, \tau \gamma_{g}\right]$ своими решениями.

Bсе такие дивизоры “допустимы”, т.е. задают вещественные неособые решения уравнения SG; при заданной $\Gamma$ мы имеем $2^{m}$ вещественных торов [1]. $\Theta$-функциональное описание этих торов [2], [3] не дает ничего для топологического заряда. Поэтому в [4] был предложен другой подход. Однако в нем выявились пробелы [5]. Здесь мы развиваем метод, дающий полное доказательство формулы, предложенной в [4] для топологического заряда.

Лемма 2. График полинома $P_{g-1}(\lambda), \lambda \in \mathbb{R}$, нигде не попадает внутрь овалов кривой $y= \pm R(\lambda)^{-1 / 2} \lambda^{-1}$. Здесь $\lambda$ таковы, что $R(\lambda) \geqslant 0$. При $\lambda>0$ мь имеем $\left|P_{g-1}(\lambda)\right| \leqslant R(\lambda)^{-1 / 2} \lambda^{-1}$. Все полиномы с этим свойством соответствуют допустимым дивизорам, причем один полином $P_{g-1}(\lambda)$ определяет $2^{g}$ допустимых дивизоров, которые получаем, выбирая из каждой пары $\left(\gamma_{j}, \tau \gamma_{j}\right)$ по одной точке произвольным образом.

"Топологический тип" полинома $P_{g-1}(\lambda)$ определяется как набор знаков $\left[s_{1}, \ldots, s m\right]$, $s_{j}= \pm 1: \quad s_{k}=1$, если графок $y=P_{g-1}(\lambda)$ проходит над овалом с номером $k$, т.е. $P_{g-1}(\lambda) \geqslant R(\lambda)^{-1 / 2} \lambda^{-1} ; s_{k}=-1$, если граффик $y=P_{g-1}(\lambda)$ проходит под овалом с номером $k$, т.е. $P_{g-1}(\lambda) \leqslant R(\lambda)^{-1 / 2} \lambda^{-1}$ для $\lambda \in\left[E_{2 k}, E_{2 k-1}\right], k=1, \ldots, m$.

Лемма 3. Все топологические типь непусты и непрерывно варьируются вместе $c$ точками ветвления кривой Г. В каждом типе множество допустимых полиномов выпукло; можнно выбрать представителя вида

$$
\begin{aligned}
P_{g-1}(\lambda)= & \sqrt{-E_{1}-E_{2 m}} \\
& \times(-1)^{s_{1}} \prod_{k=2}^{m}\left(\sqrt{E_{2 k-2} E_{2 k-1}}-(-1)^{s_{k} s_{k-1}} \lambda\right) \prod_{k=m+1}^{g}\left(\sqrt{E_{2 k-1} E_{2 k}}-\lambda\right) .
\end{aligned}
$$

ЛЕмма 4. $\lambda$-проекиии точек допустимого дивизора $\gamma_{j}=\left(\lambda_{j}, \mu_{j}\right)$ таковь, что $\lambda_{j}$ не может лежать в сегментах $\left[E_{1}, E_{0}\right],\left[E_{3}, E_{2}\right], \ldots,\left(-\infty, E_{2 m}\right]$, где $0>E_{1}>E_{2}>\cdots$.

Рассмотрим теперь "стабильный случай”, где $m=g$. Выберем базис $a$-циклов, где $a_{j}$ охватьвает отрезок между точками ветвления $E_{0}$ и $E_{2 j+1}, j=0, \ldots, g-1$. Канонический базис 
дифференциалов первого рода $\oint_{a_{j}} \omega_{k}=\delta_{j k}$ имеет вид

$$
\omega_{k}=i R(\lambda)^{-1 / 2}\left(\sum_{j=0}^{g-1} D_{j}^{k} \lambda^{j}\right) d \lambda, \quad D_{j}^{k} \in \mathbb{R}, \quad k=0, \ldots, g-1 .
$$

Теорема. Плотность топологического заряда имеет вид:

$$
2 \pi \bar{n}=\pi \sum_{k=0}^{g-1}(-1)^{k-1} s_{k} \widetilde{D}^{k}
$$

для всех конечнозонных вещественных решений уравнения $S G$ в стабильном случае, әде $\widetilde{D}^{k}=D_{g-1}^{k}+\prod_{q=1}^{2 g} E_{q}^{-1 / 2} D_{0}^{k}$.

Из свойств преобразования Абеля вытекает, что плотность топологического заряда имеет вид: $\bar{n}=\sum_{k=0}^{g-1} \widetilde{D}^{k} n_{k}$, где $n_{k}$ - аналоги топологических зарядов, отвечающие сдвигам по "временам" $t_{k}$, соответствующим базисньм циклам абелева тора. Это - целые числа, которые надо вычислить.

Удалим отрезки $\left\{\left[E_{1}, E_{0}\right],\left[E_{3}, E_{2}\right], \ldots,\left(-\infty, E_{2 m}\right]\right\}$ из $\Gamma$. После этого $\Gamma \backslash\{\cdot\}$ распадается на два взаимно несвязанных листа $G_{+}$и $G_{-}$. Для любого $g>0$ мы выберем $\Gamma$ и допустимые дивизоры $D$ в каждой компоненте так, чтобы движению по времени $t_{k}$, отвечающему циклу с номером $k$ абелева тора, соответствовало движение точки $\gamma_{k}$ только на листе $G_{+}$или $G_{-} ;$точки $\gamma_{j}, j \neq k$, движутся по стягиваемым циклам. Эти потоки нелокальны. Все вещественные решения неособы. В этих примерах мы получаем $n_{k}=(-1)^{k-1} s_{k}, n_{j}=0, j \neq k$. Здесь используются "уравнения Дубровина" для движения дивизора $D$ по указанным "временам". Меняя параметры $(\Gamma, D)$ вдоль кривой общего положения, мы будем встречать сингулярные дивизоры. Кривые $\gamma_{s}\left(t_{k}\right)$ неизбежно встретят места, где $\gamma_{j}\left(t_{k}\right)=\gamma_{s}\left(t_{k}\right)$. Однако симметричное выражение $e^{i u}=$ const $\cdot \gamma_{1} \cdots \gamma_{g}$ меняется непрерывно. Это вытекает из $\Theta$ - функциональных формул. Это и позволяет распространить формулу топологического заряда на все функциональное пространство. Использование времен $t_{k}$ и проход сингулярности играют решающую роль

В подробной работе мы приведем все детали, а также переменные действие-угол, следуя нашей схеме.

\section{СПИСОК ЛИТЕРАТУРЫ}

[1] И. В. Чередник // Докл. АН СССР. 1980. Т. 252. № 5. С. 1104-1108. [2] Б. А. Дубровин, С. М. Натанзон // Функц. анализ и его прил. 1982. Т. 16. № 1. С. 27-43. [3] N. M. Ercolani, M. G. Forest // Comm. Math. Phys. 1985. V. 99. № 1. P. 1-49. [4] Б. А. Дубровин, С. П. Новиков // Докл. АН СССР. 1982. Т. 267. № 6. С. 1295-1300. [5] С. П. Новиков // Зап. научн. семин. ЛОМИ. 1984. Т. 133. С. 177-196.

Институт теоретической Физики им. Л. Д. Ландау РАН;

Принято редколлегией University of Maryland at College Park 